\title{
The Effects of Grain Size, Oxidizers and Catalysts on Band Gap Energy of Gelam-Wood Carbon
}

\author{
Nirwan Syarif ${ }^{1,2,3^{*}}$, Dedi Rohendi ${ }^{1,2,3}$, Sri Haryati ${ }^{4}$, Claudia Kartika Sari Dewi ${ }^{5}$ \\ ${ }^{1}$ Department of Chemistry, Universitas Sriwijaya, Palembang, Indonesia \\ ${ }^{2}$ Center of Research Excellent in Fuel Cell and Hydrogen, Palembang, Indonesia \\ ${ }^{3}$ National Center for Sustainable Transportation Technology, Indonesia \\ ${ }^{4}$ Department of Chemical Engineering, Universitas Sriwijaya, Palembang, Indonesia \\ ${ }^{5}$ Undergraduate Program of Department of Chemistry, Universitas Sriwijaya, Palembang, Indonesia \\ *Email: nsyarif@unsri.ac.id
}

\begin{abstract}
The research of the effects of grain size, oxidizers, and catalysts on band gap energy of gelam-wood carbon has been conducted in which the carbons were produced from gelam-wood pyrolysis in high temperatures. The instrumentations used in this study were UV-Vis, FTIR spectrophotometer, and SEM. SEM and FTIR were used to characterize the morphology and the functionality of the carbon surface. UV-Vis spectrograms showed that the electronic property of carbon such as band gap was affected when grain size and surface area were changed. The increase of the functional groups in carbon occurred as the surface area of the carbon was increased. Band gap energy of crystalline carbon became much lower along with the increase in grain size due to the effects of bands-broadening. FTIR spectrograms showed that the carbon contained of hydroxyl and carboxylic groups. The hydroxyls were derived from steam-oxidized carbon that was provided narrower in the interlayer distance and lower-set band gap energy. Carboxylic groups were derived from acid nitric oxidation causing flat layer to become curved. The layers were wider and the band energy was higher. The main factor that affects the electronic structure of metal oxide in carbon/metal oxide composites was atomic alignments. The band gap energy increased along with the increase of the asymmetry alignments in metal oxide.
\end{abstract}

Keywords

Energy; Storage; Metal; Oxide; SEM; FTIR; UV-Vis; Composite; Gelam

\section{Introduction}

Binchotan or white carbon or white charcoal is a carbon material that is produced from biomass materials particularly hard wood from mangrove forests [1]. The application of white carbon is quite traditional, requires drinking-water purification materials, fuel, traditional health ingredients, and kitchen-tools coating mixture. Unlike the much well known black carbon (charcoals), white carbons are produced in relatively high temperatures using special woods such as gelam wood. Its unique properties were attributed from its fiber structures and compositions [2,3].

The fiber structures of the wood leads to its better physical and mechanical strength. Traditionally, the woods were pyrolyzed at $1000-1100{ }^{\circ} \mathrm{C}$ for 7 days with a very slow-rate heating in a refractory stove without using any chemicals. The product is a hard and solid greyish carbon. This pyrolysis process at this temperature leads the carbon to generate electrical conductivity properties ranging from 0.1 to $2 \mathrm{ohm}$. White carbon ability to conduct electricity makes this material potentially possible to be developed for electronic-electrical applications such as electrode materials in energy storage devices, semiconductor material of carbon dots of the DSSC, or as bipolar plates in fuel cells.

It is also necessary to study the effect of other components in carbon, i.e., oxidizer or catalyst and metal oxides towards carbon properties. Metal oxides were commonly found in carbon [4]. They exist as natural components in biomass matrices of carbon precursors or as catalysts that were added in the carbon preparation. Therefore, some metal oxide, i.e., $\mathrm{TiO}_{2}$, $\mathrm{Fe}_{2} \mathrm{O}_{3}$, and $\mathrm{NiO}_{2}$ were applied. Each of the oxides was mixed with the carbon so that metal oxide/carbon composites were prepared. Because the metal oxides were semiconductors in nature, therefore it was expected that the composites will also exhibit certain effect to the electrical properties. Electrical conduction properties appeared along with the presence of crystalline in the carbon [5].

Charge transfer process is connected by the electron cloud in the middle of hexagonal network layers of 
crystalline carbon [6]. The existence of the electron cloud is strongly influenced by the carbon network environment. When the charge transportation by electron is disturbed, the conductivity will be disrupted. Theoretically, the disturbance in the transition of electron can be interpreted as the increase of gap in the band structure of the carbon [7]. The disturbance occurs in the electron cloud due to the presence of functional groups, metallic atoms, and hybridization on crystalline of hexagonal carbon layers. Electrical property of the carbon is derived from ultraviolet (UV) spectroscopic measurements.

Experimentally, the band gap can be calculated from the UV-Vis data by using Tauc formula. By utilizing the Kubelka Munk equation, the relationship between the values of the bandgap and the reflectance can be obtained. The band gap of the carbons can be derived from regression plot of the absorption data by using direct transition equations. This paper notifies the effects of the grains size, the oxidizing agents and the type of the transition metals to the band gaps of carbon material. These relationships were generated from UVVis measurement data [8]. The existence of a transition metal and the functional groups in the carbon were detected by using SEM - EDX and FTIR respectively [9].

\section{Methodology}

\subsection{Carbon preparation}

The starting materials were prepared by using gelam carbon block and gelam wood powder. Gelam wood was derived from the carpentry and wood industry which was obtained from logs with a diameter of 8-10 $\mathrm{cm}$. Gelam wood was separated from its bark and was cleaned with flowing clean water and was removed from organic impurities with hot water. The gelam wood was cut using an electric saw and rinsed with tap water. Then it was let dry for 24 hours and sieved in 100 mesh to produce Gelam Wood Sawdust (GWS).

GWS was soaked in a solution of $20 \% \mathrm{HCl}$ for 1 day to remove the existing inorganic residue. Then, the inorganic-residue free GWS was dried in an oven at a temperature of $110{ }^{\circ} \mathrm{C}$ for 24 hours. The cleaned and dried GWS was dipped in ethanol metallic salt solution of $\mathrm{Fe}, \mathrm{Ti}$, and $\mathrm{Ni}$ for 16 hours. Then, the mixture of GWS and the solution were re-dried in the oven. After being dried, the dried powders were pyrolyzed at nitrogen environment for 12-13 hours in an electrical furnace. The flow rate was $1 \mathrm{~mL}$ minute $^{-1}$ and the temperature raised by $3{ }^{\circ} \mathrm{C}$ per minute. The temperature inside the furnace was kept stable at $1100^{\circ} \mathrm{C}$ for 1 hour before the carbon was allowed to cool off inside the furnace with a temperature rate of $3{ }^{\circ} \mathrm{C}$ per minute. The products were GWCN for GWCNTi, GWCNFe, and GWCNNi powder.

Gelam carbon blocks were made of gelam wood blocks using repetitive pyrolysis technique. Gelam wood blocks were heated at a temperature of $260{ }^{\circ} \mathrm{C}$ for 0.5 hour to remove any adsorbed water molecules that existed in the matrices of the wood to be able to produce a material known as torrefied wood. To produce carbon, the torrefied wood was pyrolyzed for 0.5 hours at 1100 ${ }^{\circ} \mathrm{C}$ before being cooled off. The pyrolysis process was repeated once more with the same temperature and duration to produce gelam carbon block. The carbon was labelled as CCP.

Surface oxidation process was proceeded to two parts of CCP using concentrated nitric acid and high-pressure steam. The products were respectively labelled as $\mathrm{CCPHNO}_{3}$ and CCPWOT. CCP was oxidized in reflux system for 3 hours using concentrated nitric acid at a temperature of $60^{\circ} \mathrm{C}$ to produced $\mathrm{CCPHNO}_{3}$. The highpressure steam oxidation was proceeded in the hydrothermal reactor at a temperature of $200{ }^{\circ} \mathrm{C}$ for 16 hours. Then, the products were washed with abundant demineralized water and dried to produce CCPWOT.

\subsection{Characterization}

Changes appeared in the functional groups after the oxidation process was performed with the transition of metal oxides as catalysts to prepare the carbons and electronic properties of carbons. The chages were characterized by respectively using FTIR, SEM-EDX, and UV-Vis instrumentations. FTIR spectrogram were obtained by using Shimadzu Prestige 21 that were carried out on the diffuse reflectance mode. UV-Vis spectrogram were obtained by using Shimadzu UV2450. Boehm titration was used to support analyses of surface functionality of carbon. The procedure for the titration can be found elsewhere [10]. The image of morphology of the surface and elemental composition of the carbon samples were obtained by using SEMEDX JEOL JSM 9390A.

Characterization for electronic property of the carbons was conducted in Shimadzu UV-Vis spectrophotometer UV-2450 with wavelength ranges from 200 to $800 \mathrm{~nm}$ in diffuse reflectance mode. The energy band gap can be generated by using the Kubelka-Munk function on the UV-Vis test data (spectrogram). Kubelka-Munk function is stated in equation (1).

$$
F(R)=\left(\frac{(1-R)^{2}}{2 R}\right)
$$


Where $\mathrm{R}$ is the reflectance of carbon sample that was obtained from UV-Vis spectrogram. The values of $F(R)^{2}$ were plotted against the result of wavelengths conversion, i.e., energy values by using the equation that is stated in equation (2).

$$
E=h \frac{c}{\lambda}
$$

Where $E$ is the band gap energy; $h$ is Plank constant value $\left(6.6261 \times 10^{-34} \mathrm{Js}\right)$; $\mathrm{c}$ is the value of speed of light in the air $\left(3 \times 10^{8} \mathrm{~ms}^{-1}\right)$ and $\lambda$ is wavelength. The Kubelka-Munk equation (1) is substituted into Tauc equation which described the relationship between the absorption coefficient and band gap energy. The Tauc equation is stated in equation (3).

$$
(h v \alpha)^{1 / n}=h v-E_{g}
$$

Where $\alpha, v, A$ and $E_{g}$ respectively are values of absorption coefficient, light frequency, proportionality constant, and band gap. The absorption coefficient in equation (3) can be replaced by Kubelka-Munk function (1) therefore, the equation can be written as equation (4).

$$
(h v F(R))^{1 / n}=h v-E_{g}
$$

The value of the power (n) denotes the nature of the electron transition in the carbon. The $1 / 2$ is for direct transition, and the 2 is for indirect transition. In this study, it is assumed that there is no photon involved, therefore the plot is $(h v F(R))^{2}(\mathrm{huF}(\mathrm{R}))^{2}$ versus $h v$. Extrapolation of linear part of the curve cross the $\mathrm{X}$-axis which makes $(h v F(R))^{2}=0$, or axis intercepts and gives band gap energy of the carbon.

\section{Results and Discussion}

The grain size is an important factor that can affect the characteristic and application of carbon $[11,12]$. The changes in particle size will cause changes on the surface. The surface of carbon is the place where the variety of functional groups is attached. The change that occurred to the surface functionality of the carbon as the function of the particle size can be seen in FTIR spectrograms (Figure 1).

It can be shown that spectrograms are relatively similar at the wavenumbers below $750 \mathrm{~cm}^{-1}$. It can be interpreted that there was no change especially for vibration bonds between carbon and inorganic elements or single bonds carbon atoms. The increase in transmittance can be interpreted as the increase of the functional groups which is the result of the increase of the surface area of the carbon. This indication can be related to the excellent absorption property of carbon. Therefore the formation of new surface will be responded by the volatiles and the organics to bind with the functionality on carbon hexagonal network structure.

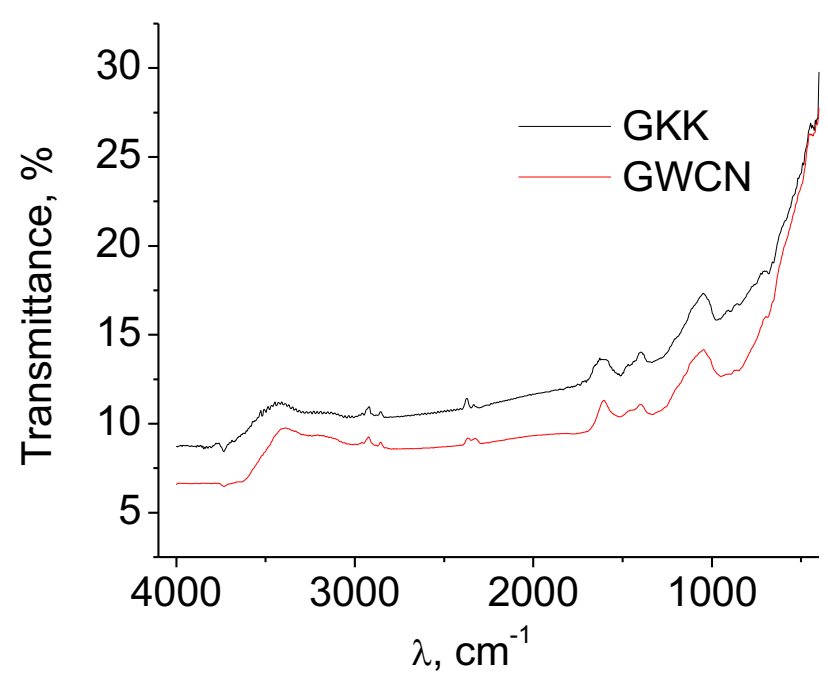

Figure 1 FTIR Spectrograms of gelam-wood carbon block (CCP) and carbon powder (GWCN).

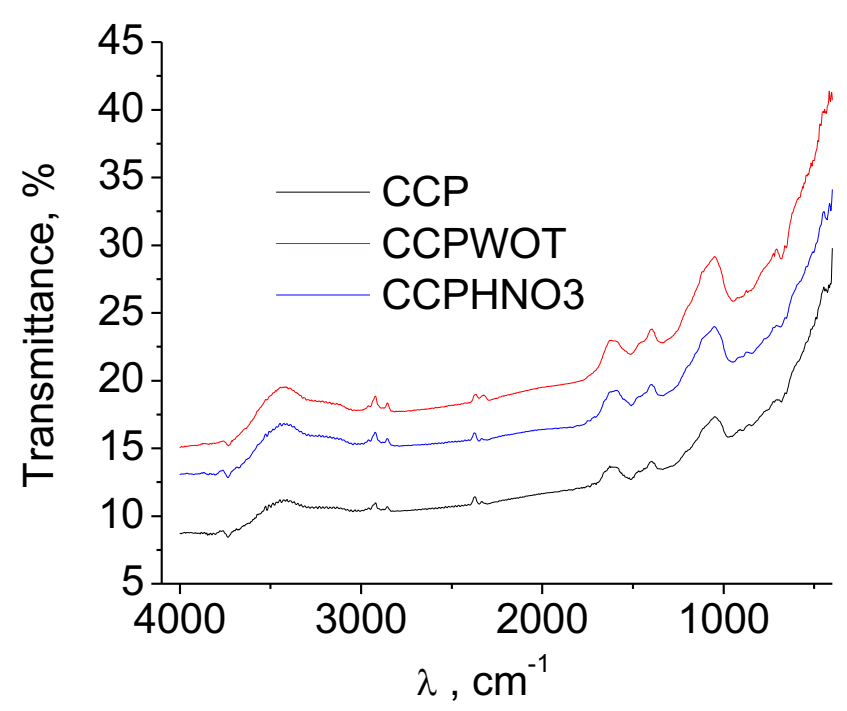

Figure 2 FTIR spectrograms of oxidized and unoxidized carbon blocks. CCP represented unoxidized, $\mathrm{CCPHNO}_{3}$ represented nitric acid oxidized carbon and CCPWOT represented steam oxidized carbon. 
Table 1 Functional Groups Composition was determined by using Boehm Titration

\begin{tabular}{cccccc}
\hline Samples & Fenolic & Lactonic & Carboxylic & $\begin{array}{c}\text { Total } \\
\text { Acid }\end{array}$ & $\begin{array}{c}\text { Total } \\
\text { Base }\end{array}$ \\
\hline $\mathrm{CCP}$ & 0.200 & 3.85 & 0.00 & 4.05 & 0.24 \\
$\mathrm{CCPHNO}_{3}$ & 0.100 & 3.08 & 0.20 & 3.38 & 0.19 \\
$\mathrm{CCPWOT}$ & 0.200 & 3.40 & 0.10 & 3.70 & 0.22 \\
\hline
\end{tabular}

The influence of oxidizing treatment to the IR spectra can is shown in Figure 2. It implies that the oxidation treatment does not bring significant change. The spectrogram shows that the oxidation cause the transmittance to increase in three regions due to the oxidation, with both water vapor and nitric acid. The $1300-1400 \mathrm{~cm}^{-1}$ is the vibrational wavenumber region for the carbonate group [8] that is connected to $1700 \mathrm{~cm}^{-}$ ${ }^{1}$ which is the vibrational wavenumber region typically for the carbonyl group. The transmittance at a wavenumber of 1500 to $1900 \mathrm{~cm}^{-1}$ corresponds to the transmittance values in the region of 2300 to $2400 \mathrm{~cm}^{-1}$ and 2850 to $2900 \mathrm{~cm}^{-1}$. The region of 2300 to $2400 \mathrm{~cm}^{-}$ ${ }^{1}$ is the vibrational wavenumber region for the $\mathrm{C}-\mathrm{H}$ or $\mathrm{CH}_{2}$ - groups, while the region of 2850 to $2900 \mathrm{~cm}^{-1}$ is the vibrational wavenumber region for the $\mathrm{C}-\mathrm{H}$ or $-\mathrm{CH}_{2}-$ groups [9].

The peaks at 2850 to $3000 \mathrm{~cm}^{-1}$ indicated the existence of carboxylate functional groups. These changes were significantly detected and confirmed by Boehm titration. The results of Boehm titration are summarized in Table 1. The calculation implies that changes occur to the concentration of the carbon functional group after the oxidation process specifically to water favor and nitric acid.

The effect of the addition of metal salt on the carbon functionality is shown in Figure 3. The spectrograms show that the catalysts affected $\mathrm{C}-\mathrm{O}-\mathrm{C}$ or carbonate groups $\left(1250 \mathrm{~cm}^{-1}\right)$ and carbonyl groups $\left(1700 \mathrm{~cm}^{-1}\right)$ on the surface of the carbon. Symmetrical $\mathrm{C}=\mathrm{C}$ bond on the carbon surface can be suppressed by adding $\mathrm{TiO}_{2}$. The increase of peak intensity around $3600 \mathrm{~cm}^{-1}$ inferred the increase of $\mathrm{OH}$ functionality. This feature is in sync with the increase of $\mathrm{C}=\mathrm{O}$ showed by the peak around $1700 \mathrm{~cm}^{-1}$ for the carbon with the catalysts of $\mathrm{Ni}$ (GWCNNi) and Ti (GWCNTi). In other words, Ni and Ti salts tend to increase the carboxylate on the carbon. The same trend is shown in the absorption region of $\mathrm{C}$ $-\mathrm{C}$ and $\mathrm{C}=\mathrm{C}$, where the use of metal salts $(\mathrm{Ni}$ and $\mathrm{Fe}$ ) as activators decrease the intensity of IR. The different tendencies are shown in $\mathrm{C}-\mathrm{O}$ and $\mathrm{C}=\mathrm{C}=\mathrm{O}$ which are the representation of phenolic and lactonat. Furthermore, the identification of the functional group composition can explain the changes on the surface of activated carbon.

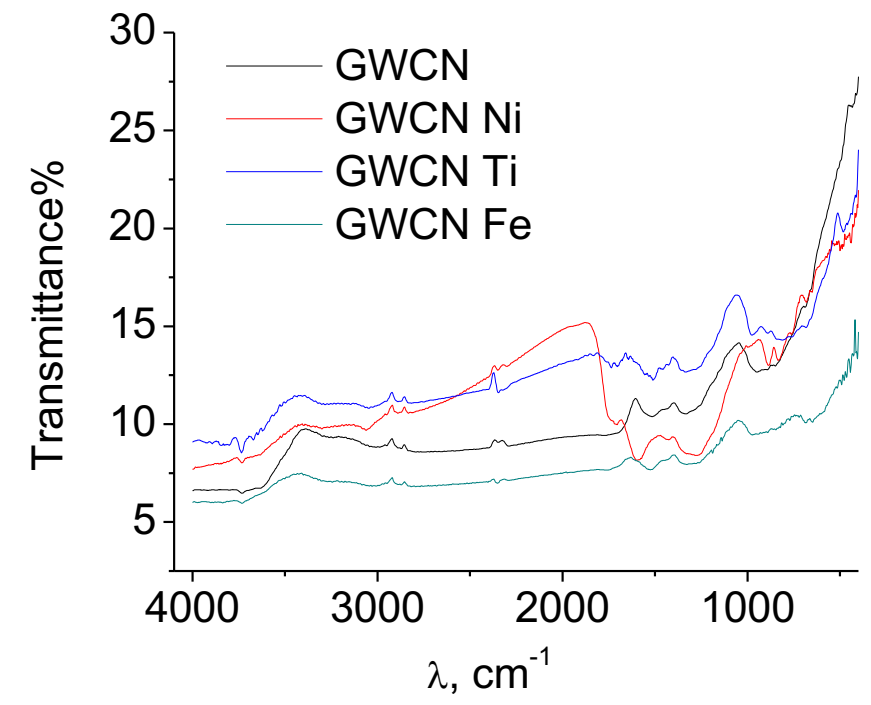

Figure 3 FTIR Spectrograms of uncatalyzed and catalyzed carbon powders oxidized and unoxidized carbon blocks, i.e., uncatalyzed carbon $(\mathrm{GWCN})$, catalyzed carbon powder with metal salt of $\mathrm{Ni}$, Ti, and Fe, i.e., GWCNNi; GWCNTi and GWCNFe.

SEM images (Figure 4) show that there is no significant difference in the morphology of the metal catalyzed carbons which represent three different types of carbons. Microstructures of the carbons powder are featured with tiny fragment of 10-30 $\mu \mathrm{m}$ in irregular shape. However, the differences can be found in SEM images of carbon at 1000x magnification. The two of four carbons show that their fragments have some layers.

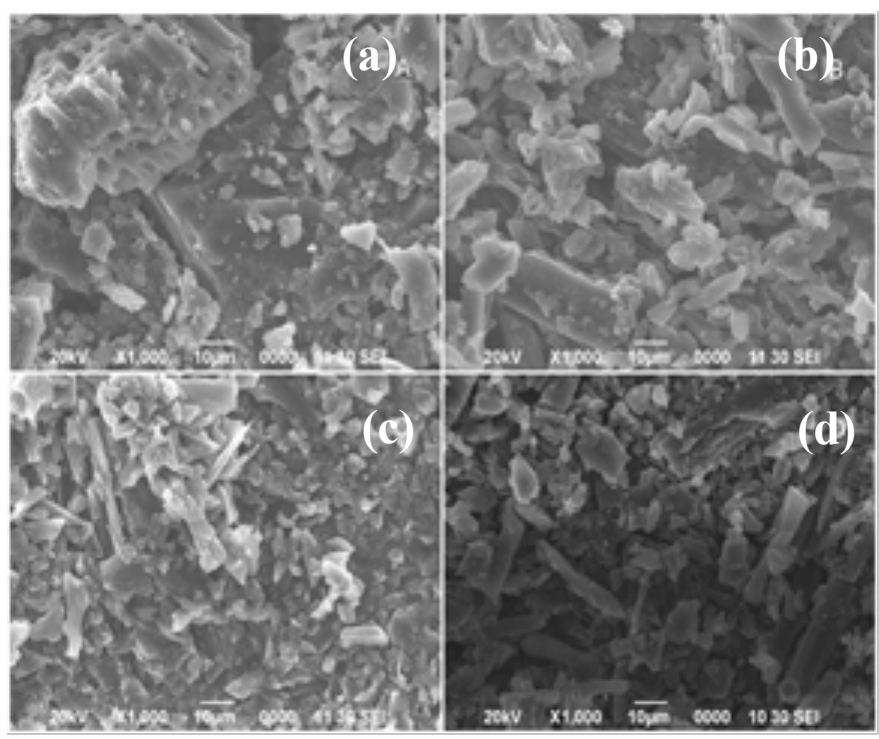

Figure 4 SEM Images of carbons at 1000x magnification for (a) prepared without catalyst, (b) with $\mathrm{Fe}$ catalyst (GWCNFe), (c) with Ti catalyst (GWCNTi) and (d) with Ni catalyst (GWCNNi). 


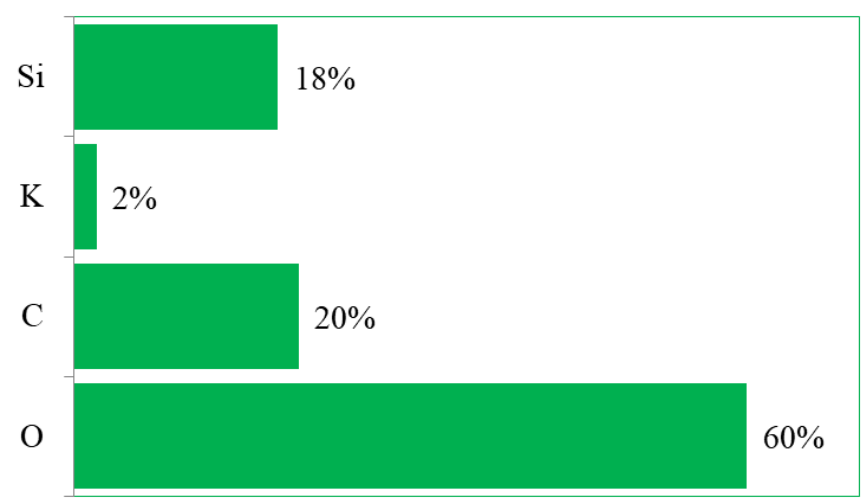

Figure 5 EDX spectra of carbon without catalyst

EDX analyses of gelam-wood carbon (Figure 5) show that the carbon contained $18-22 \%$ of silica. Silica can be observed using an electron microscope which appears as a bright spot as it tends to look lighter in carbon. The existence of silica in the carbon matrices were quite the same as the morphological properties of $\mathrm{C} / \mathrm{SiO}^{2}$ composites. The composites are known to have semiconductor properties [13, 14]. Therefore, in order to have a detail explanation about the existence of $\mathrm{SiO}_{2}$ in gelam-wood carbon conductivity, a set of experiments was conducted in laboratory.

EDX analyses results (Figure 6) showed that the composite samples contained $4.38 \%$ of Fe in GWCNFe (Figure $6(\mathrm{a})$ ), $6.83 \%$ of Ti in GWCNTi (Figure 6 (b)) and $5.44 \%$ of Ni in GWCNNi (Figure 6 (c)). It can be seen that the EDX data shows the differences in metaloxides content in the carbon samples, i.e, GWCNFe, GWCNTi and GWCNNi. The actual content of metal oxides wererelatively low compared to the initial content. Initially, the metal-contents were 5\%,7\% and $6 \%$ for GWCNFe, GWCNTi and GWCNNi. In such conditions, it was expected that the bias of the interpretation of the relationship between the metal content of the electronic properties of carbon is low.

As shown in Figure 7, the wavelength of maximum absorption was observed in $350-500 \mathrm{~nm}$ (Figure 7 (a)) which means that the electronic $\pi \rightarrow \pi^{*}$ transition occurred in a conjugated carbon system [15]. It is also interpreted that the carbon absorbed the ultraviolet that was near to the visible region of electromagnetic spectrum. The UV spectra of carbon block has other maximum absorption located further in the ultraviolet region $\left(\sigma \rightarrow \sigma^{*}\right)$ with low intensity.

The transition has the band gap energies (Figure 7 (b)) of $2.30 \mathrm{eV}$ for the carbon block (CCP), and $2.25 \mathrm{eV}$ for the carbon powder (GWCN). Band gap energy of crystalline carbon gets lower as the grain size gets bigger due to the effects of bands-broadening. In a single atom, the band gap is equal to the distance between the ground level (HOMO) and the first excited level (LUMO). With the increasing number of atoms, both levels are broadened. This broadening leads the band gap to be narrow.

The effect of the surface functionality to the band gap energy can be derived from FTIR spectra of oxidized carbons. Steam oxidized carbon (CCPWOT) provided $\mathrm{OH}$ group which dominates the carbon surface, whilst the nitric acid oxidized carbon $\left(\mathrm{CCPHNO}_{3}\right)$ provided much more carboxylic groups compared to how much the CCP has [16]. The wavelength of maximum absorption was observed in 350-500 $\mathrm{nm}$ and $200-250$ $\mathrm{nm}$ (Figure 8 (a)) for electronic $\pi \rightarrow \pi^{*}$ transitions in unoxidized and oxidized carbon. The plots suggested that the oxidizers had small effect on conjugated hexagonal frame of carbon, instead of attaching new surface functionalities.
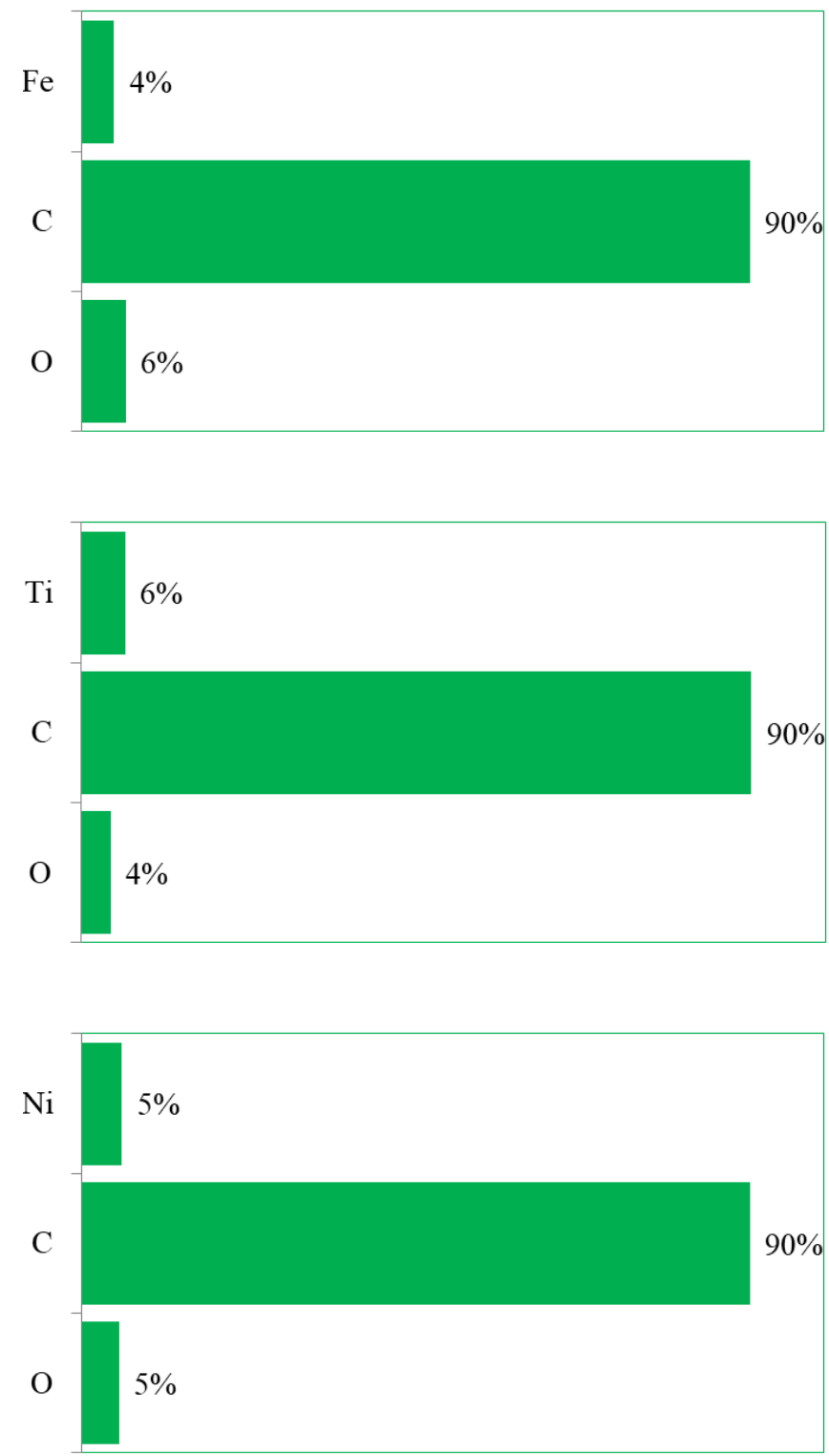

Figure 6 EDX spectra of carbon with catalyst 
Band gap energies (Figure 8 (b)) for $\mathrm{CCPHNO}_{3}$, CCPWOT and CCP respectively were $1.25 \mathrm{eV}, 2.75 \mathrm{eV}$ and $2.30 \mathrm{eV}$. The change occurred due to sensitivity of lattice symmetry of the carbon layer structures. By controlling the carrier density between layers, the occupation of electronic states near the Fermi level, and the magnitude of the gap between the valence and conduction bands can be manipulated. The functionality might be induced by an electric field across the layers, i.e., carboxylic groups in carbon cause flat layer to curve and the distance between layer becomes wider. A widened interlayer will generate a wider band gap energy. Conversely, hydroxyl groups cause the interlayer distance to be narrower [17] and it set a lower band gap energy.
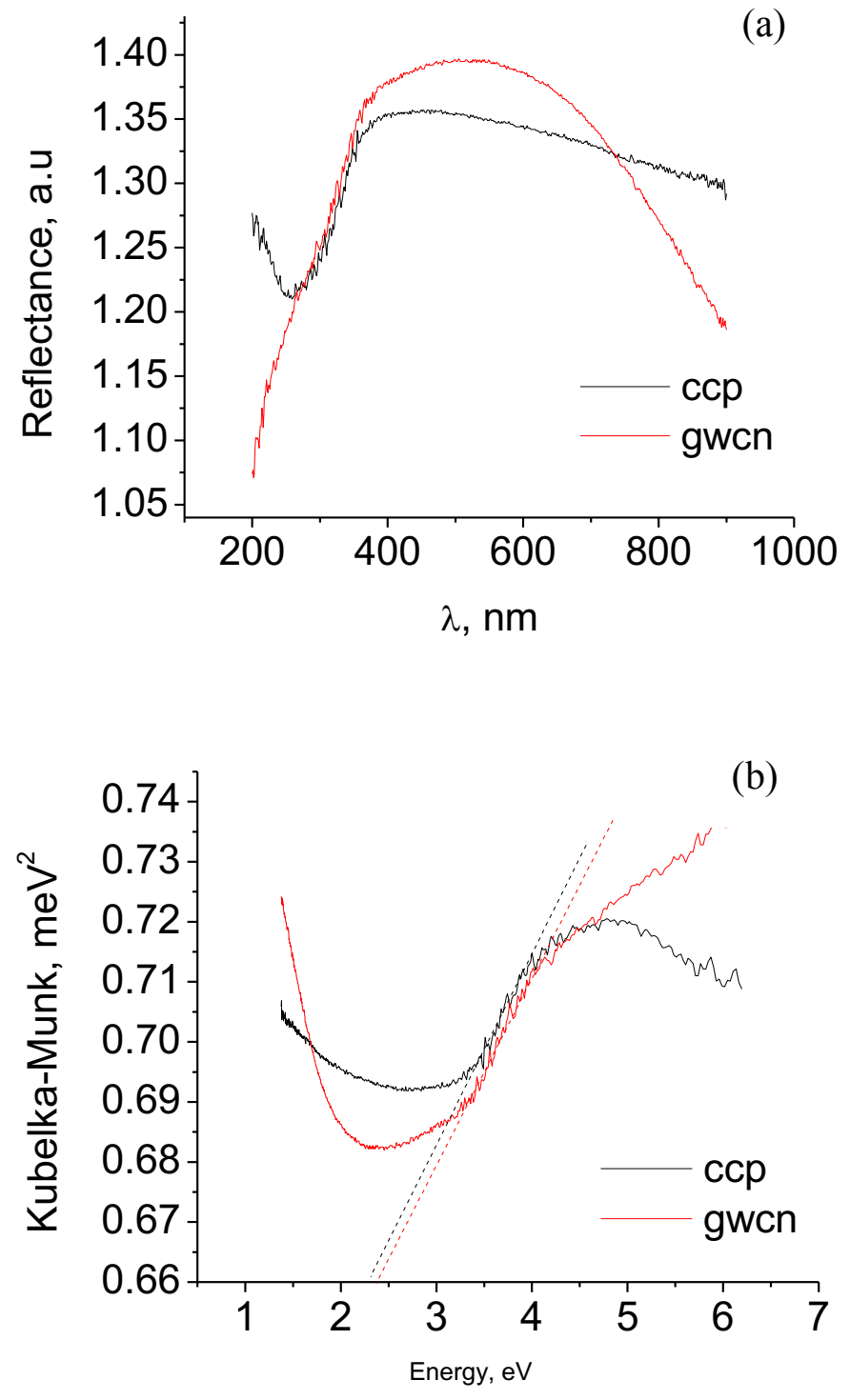

Figure 7 (a) UV-Vis Spectra (b) Tauc Plot of Kubelka-Munk vs Energy for two types of carbons, GWCN and CCP for powder and block carbon, respectively.
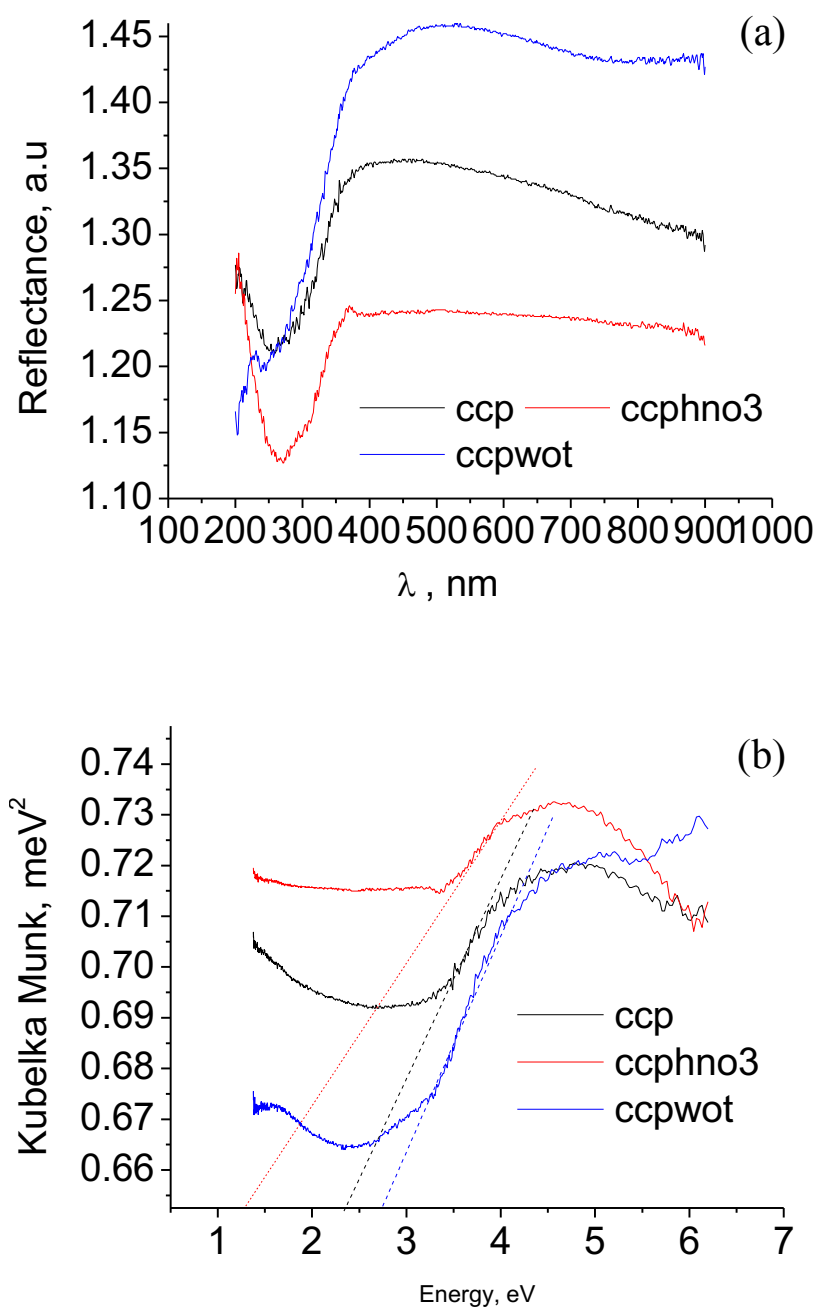

Figure 8 (a) UV-Vis Spectra (b) Tauc Plot of Kubelka-Munk vs. Energy for unoxidized and oxidized carbons, CCP, CCPHNO3 and CCPWOT for unoxidized, acid nitric-oxidized and wateroxidized carbon, respectively.

The effect of metal oxides to the electronic transitions of carbons is shown in spectrograms (Figure 9 (a)). The curves experience its peaks near the ultraviolet - visible region of electromagnetic spectrum. The addition of metal oxides in carbon matrices pushed the peaks to shift slightly to the shorter wavelength.

The dependences of $(h v F(R))^{2}(\mathrm{~h} v \mathrm{~F}(\mathrm{R}))^{2}$ versus $h v$ that are obtained for the non-catalyzed and catalyzed carbons are shown in Figure 9 (b). The extrapolation of the linear part of the curve for the non-catalyzed carbon obtained the direct band gap of $2.50 \mathrm{eV}$, whilst the band gap energies (Figure 9 (b)) for GWCNFe, GWCNTi and GWCNNi are $3.00 \mathrm{eV}, 1.00 \mathrm{eV}$, and $2,25 \mathrm{eV}$. The metal oxides existed in the carbon as composites. Therefore, the electronic properties of metal oxide can be explained as a part of the whole electronic process in the composites. 

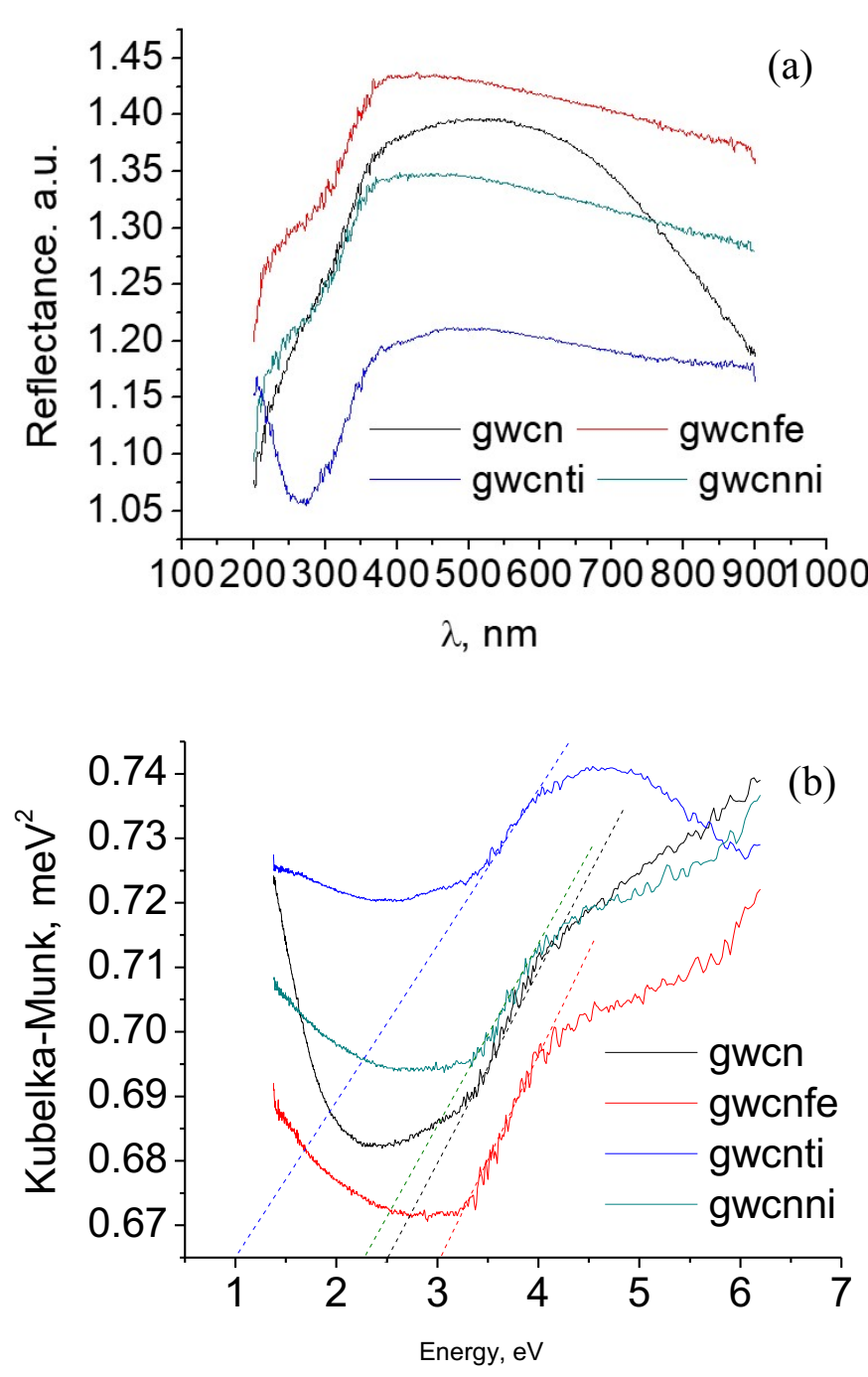

Figure 9 (a) Spectra UV-Vis (b) Plot KubelkaMunk vs Energy for the carbon that were catalyzed by the addition of transition-metal salt on carbon powder and non-catalytic carbon.

The main factor that affects the electronic structure of metal oxide is the atomic alignments [18]. The alignments can be described as an approximately closepacked arrangement of oxygen ions with metal ions that are distributed respectively among two kinds of interstice which are tetrahedral and octahedral coordination sites. The eightfold position is the tetrahedral sites, and the sixteenfold position is the octahedral sites. The lack of symmetry in atomic alignments at the interfaces would cause lack of relaxation, develop defects and distort the chargetransfer. $\mathrm{Fe}_{2} \mathrm{O}_{3}$ and $\mathrm{NiO}$ are the oxides that have very low deviations from the cubical atomic symmetry at low temperatures $[19,20]$. The band gaps energy will increase along with the increase of the asymmetry alignments in metal oxide [11]. Inversely, $\mathrm{TiO}_{2}$ has a high asymmetrical in atomic alignments [21], therefore the band gap energy is relatively high compared to the others.

\section{Conclusion}

Basic effect on carbon that occurred when the particle size is changed is the surface area. The surface area is the place where varieties of functional groups are attached. The functional groups in carbon experienced an increase because of the increase of the surface area of the carbon. Band gap energy of crystalline carbon gets lower as the grain size gets bigger due to the effects of bands-broadening.

Surface functionality may affect the band gap energy. Hydroxyl functionality is derived from steam oxidized carbon that provides a narrower interlayer distance and a lower band gap energy. Carboxylic groups are derived from acid nitric oxidation that cause the flat layer to become curved, the distance between layers becomes wider and the band gap energy becomes higher. The metal oxides exist in carbon as composite, therefore, electronic properties of metal oxide can be explained as a part of the whole electronic process in the composites. The main factor that affects the electronic structure of metal oxide is the structure of atomic alignments in molecule. $\mathrm{Fe}_{2} \mathrm{O}_{3}$ and $\mathrm{NiO}$ are the oxides that have very low deviations from the cubic symmetry at low temperatures. The band gap energy will increase along with the increase of the asymmetry alignments in metal oxide. Inversely, $\mathrm{TiO}_{2}$ has high asymmetrical in atomic alignments, therefore the band gap energy is relatively high.

\section{Acknowledgment}

The financial support for this research provided by Dikti and DRPM UI (contract number: 409u/DRPM$\mathrm{UI} / \mathrm{A} / \mathrm{N} 1.4 / 2009$ ) is gratefully acknowledged for the award of a Doctorate grant. This paper is supported by USAID through Sustainable Higher Education Research Alliances (SHERA) Program - Center for Collaborative (CCR) National Center for Sustainable Transportation Technology (NCSTT) with grant no. IIE00000078-ITB-1.

\section{References}

[1] A. Goessens et al., "Is Matang Mangrove Forest in Malaysia Sustainably Rejuvenating after More than a Century of Conservation and Harvesting Management?," PLOS ONE, vol. 9, no. 8, p. e105069, Aug. 2014, doi: 10.1371/journal.pone.0105069.

[2] K. L. Spence, R. A. Venditti, Y. Habibi, O. J. Rojas, and J. J. Pawlak, "The effect of chemical composition on microfibrillar cellulose films from wood pulps: Mechanical processing and physical properties," Bioresource Technology, vol. 101, no. 15, pp. 5961-5968, Aug. 2010, doi: 10.1016/j.biortech.2010.02.104.

[3] M. Salajkova, L. Valentini, Q. Zhou, and L. A. Berglund, "Tough nanopaper structures based on cellulose nanofibers and carbon nanotubes," Composites Science and Technology, vol. 87, pp. 103-110, Oct. 2013, doi: 10.1016/j.compscitech.2013.06.014. 
[4] N. Syarif, A. M. Mahmuda, S. Haryati, E. Yunita, W. Sudarsono, and C. T. Lee, "Application of water lettuce (pistia s.) conductive carbon in electrochemical capacitor," Chemical Engineering Transactions, pp. 499-504, 2018005, doi: 10.3303/CET1863084.

[5] N. Syarif, D. Rohendi, Wulandhari, and I. Kurniawan, "Optimization of biomass-based electrochemical capacitor performance," presented at the THE 3RD INTERNATIONAL SEMINAR ON CHEMISTRY: Green Chemistry and its Role for Sustainability, Surabaya, Indonesia, 2018, p. 020057, doi: 10.1063/1.5082462.

[6] W. Sudarsono et al., "Noble- free oxygen reduction reaction catalyst supported on Sengon wood ( Paraserianthes falcataria $L$. ) derived reduced graphene oxide for fuel cell application," Int J Energy Res, vol. 44, no. 3, pp. 17611774, Mar. 2020, doi: 10.1002/er.5015.

[7] C. Xu, P. A. Brown, and K. L. Shuford, "Strain-induced semimetal-to-semiconductor transition and indirect-todirect band gap transition in monolayer 1T-TiS 2," $R S C$ $A d v$., vol. 5, no. 102, pp. 83876-83879, 2015, doi: 10.1039/C5RA16877E.

[8] N. F. Syabania, N. Syarif, D. Rohendi, M. Wandasari, and W. D. Rengga, "The Light Transmittance and Electrical Conductivity Properties of Gelam Wood Carbon Nanosheet and Its Derivatives," Indo. J. of Fund. and Appl. Chem., vol. 4, no. 3, pp. 126-131, 2019.

[9] N. F. Syabania, W. Sudarsono, D. Rohendi, and N. Syarif, "Functionality Analysis of Carbon Nanosheet, Oxidized Carbon Nanosheet and Reduced Carbon Nanosheet Oxide by Using Fourier Transform Infra Red and Boehm Titration Method," J. Phys.: Conf. Ser., vol. 1095, p. 012028, Sep. 2018, doi: 10.1088/1742-6596/1095/1/012028.

[10] H. P. Boehm, "Some aspects of the surface chemistry of carbon blacks and other carbons," Carbon, vol. 32, no. 5, pp. 759-769, Jan. 1994, doi: 10.1016/0008-6223(94)90031-0.

[11] W. F. Lim and K. Y. Cheong, "Effects of Post-Deposition Annealing Temperature on Band Alignment and Electrical Characteristics of Lanthanum Cerium Oxide on 4H-SiC," MRS Proceedings, vol. 1433, Jan. 2012, doi: 10.1557/opl.2012.1144.

[12] D. Segets et al., "Determination of the Quantum Dot Band Gap Dependence on Particle Size from Optical Absorbance and Transmission Electron Microscopy Measurements,"
ACS Nano, vol. 6, no. 10 , pp. 9021-9032, Oct. 2012, doi: 10.1021/nn303130d.

[13] T. S. Bispo, G. B. Barin, I. F. Gimenez, and L. S. Barreto, "Semiconductor carbon composite from coir dust and sepiolite," Materials Characterization, vol. 62, no. 1, pp. 143-147, Jan. 2011, doi: 10.1016/j.matchar.2010.10.010.

[14] M. T. S. Chani et al., "Temperature Gradient Measurements by Using Thermoelectric Effect in CNTs-Silicone Adhesive Composite," PLoS ONE, vol. 9, no. 4, p. e95287, Apr. 2014, doi: 10.1371/journal.pone.0095287.

[15] K. K. Kim, S. M. Kim, and Y. H. Lee, "Chemically Conjugated Carbon Nanotubes and Graphene for Carrier Modulation," Accounts of Chemical Research, vol. 49, no. 3, pp. 390-399, Mar. 2016, doi: 10.1021/acs.accounts.5b00441.

[16] C. Liu and G. Hu, "Effect of Nitric Acid Treatment on the Preparation of Graphene Sheets by Supercritical N, N Dimethylformamide Exfoliation," Industrial \& Engineering Chemistry Research, vol. 53, no. 37, pp. 14310-14314, Sep. 2014, doi: 10.1021/ie5019707.

[17] H. K. Jeong, M. H. Jin, K. P. So, S. C. Lim, and Y. H. Lee, "Tailoring the characteristics of graphite oxides by different oxidation times," Journal of Physics D: Applied Physics, vol. 42, no. 6, p. 065418, Mar. 2009, doi: 10.1088/0022$3727 / 42 / 6 / 065418$.

[18] W. Qin, J. Hou, and D. A. Bonnell, "Effect of Interface Atomic Structure on the Electronic Properties of Nano-Sized Metal-Oxide Interfaces," Nano Letters, vol. 15, no. 1, pp. 211-217, Jan. 2015, doi: 10.1021/nl503389b.

[19] A. M. van Diepen and Th. J. A. Popma, "Temperature dependence of the hyperfine field in amorphous Fe2O3," Solid State Communications, vol. 27, no. 2, pp. 121-125, Jul. 1978, doi: 10.1016/0038-1098(78)90814-1.

[20] D. Su, M. Ford, and G. Wang, "Mesoporous NiO crystals with dominantly exposed $\{110\}$ reactive facets for ultrafast lithium storage," Scientific Reports, vol. 2, Dec. 2012, doi: 10.1038/srep00924.

[21] H. Anh Huy, B. Aradi, T. Frauenheim, and P. Deák, "Comparison of $\mathrm{Nb}$ - and Ta-doping of anatase $\mathrm{TiO} 2$ for transparent conductor applications," Journal of Applied Physics, vol. 112, no. 1, p. 016103, 2012, doi: $10.1063 / 1.4733350$ 